\title{
Trends In Acoustic Neuroma Management: A 20-Year Review of the Oxford Skull Base Clinic
}

\author{
Samuel A. C. MacKeith ${ }^{1} \quad$ Richard S. Kerr ${ }^{2}$ Chris A. Milford ${ }^{1}$ \\ ${ }^{1}$ Department of ENT, John Radcliffe Hospital, Oxford, United Kingdom \\ ${ }^{2}$ Department of Neurosurgery, John Radcliffe Hospital, Oxford, \\ United Kingdom \\ Address for correspondence Samuel A.C. MacKeith, MB ChB, FRCS, \\ Department of ENT, John Radcliffe Hospital, Oxford OX3 9DU, United \\ Kingdom (e-mail: samuelmackeith@doctors.org.uk).
}

J Neurol Surg B 2013;74:194-200.

\begin{abstract}
Keywords

- acoustic neuroma

- management

- trend

- radiosurgery

- vestibular schwannoma

Objective To describe the change in the management of acoustic neuromas at one United Kingdom center over a 20-year period and to compare this with what is known regarding trends in practice on a national and international scale.

Design, Setting, and Participants Data was collected prospectively on all patients attending the Oxford Skull Base Clinic between 1990 and 2009.

Main Outcome Measures The proportion of patients managed initially by observation versus radiotherapy versus surgery was recorded for each year.

Results Significantly more patients received radiation treatment (instead of surgery) between 2000 and 2009 when compared with 1990 to 1999. Compared with national audit data, the Oxford Skull Base Clinic treats a higher proportion of patients with radiotherapy and significantly lower proportion with surgery, though the trend nationally is toward more observation and radiotherapy and less surgery.

Conclusion Surgery will remain crucial in the management of some patients with acoustic neuromas (usually those with the larger tumors where radiosurgery is recognized to be less appropriate), but using current trends to predict future practice would suggest that alternative nonmicrosurgical treatment may play an increasingly important role in the future.
\end{abstract}

\section{Introduction}

Over the past 100 years, major advances have been made in the surgical management of patients with acoustic neuromas. Although significant progress was made in the management of this condition during the "Cushing era" (1900 to 1917) and the "Dandy era" (1917 to 1961), it was not until earlier detection of tumors using computed tomography (CT), and the introduction of the operating microscope, that the morbidity and mortality of this type of surgery began to fall. ${ }^{1}$

The subsequent development of magnetic resonance imaging (MRI) scanning has allowed for detection of smaller tumors as well as allowing accurate size measurements, leading to increased knowledge regarding the natural history and growth patterns of these tumors.

received

July 14,2012

accepted after revision

January 20, 2013

published online

April 1, 2013

The development of gamma knife (GK) radiosurgery, despite being first performed in 1969, has only been adopted on a more widespread scale over the past 20 years. Improved outcomes have continued with both microsurgical and GK radiosurgical techniques, and the debate in the literature regarding the relative advantages of each continues.

The wider acceptance of GK radiosurgery in the United Kingdom has further complicated the clinician's decisionmaking process when managing a patient with an acoustic neuroma. The decision to operate, give GK radiosurgery, or simply observe must be made on a case-by-case basis. However, it is clear that practice varies considerably throughout the world and even nationally between regional centers. This study describes the changes in the practice of acoustic

(c) 2013 Georg Thieme Verlag KG Stuttgart · New York
DOI http://dx.doi.org/ 10.1055/s-0033-1342919. ISSN 2193-6331. 


\section{Acoustic Neuroma Patients Per Year}

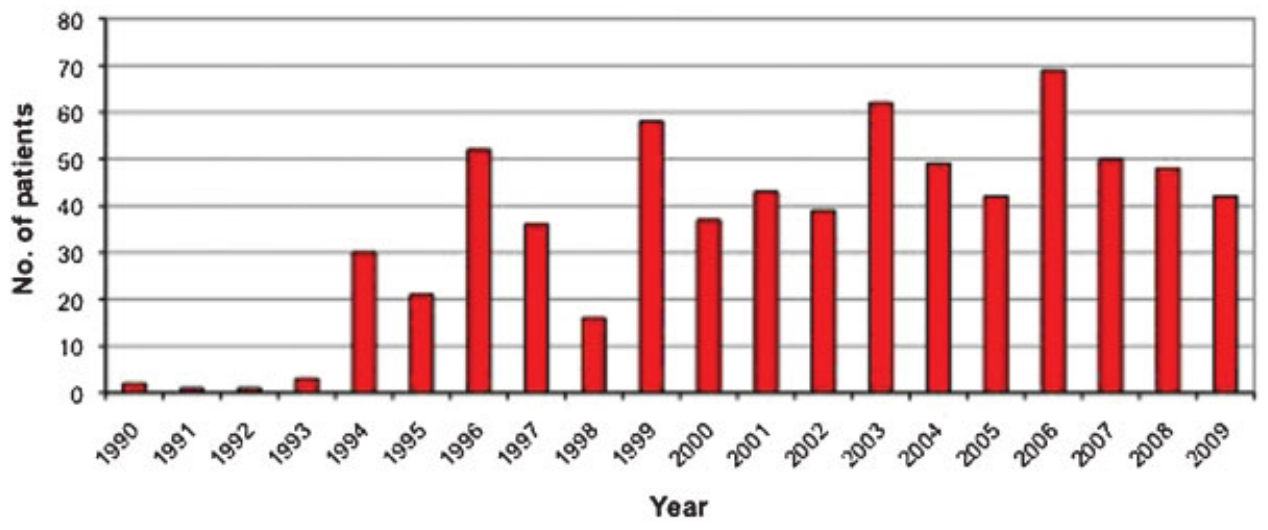

Fig. 1 Number of patients with an acoustic neuroma referred per year.

neuroma management in one center over a 20 -year period. A comparison is made with what is known regarding trends in practice on a national and international level.

\section{Methods}

Data was collected prospectively on all patients attending the Oxford Skull Base Clinic (OSBC) between 1990 and 2009. This combined neurosurgical/otolaryngological (ENT) service was established as a multidisciplinary clinic in 1990 (coinciding with the time of new consultant appointments in both the neurosurgical and ENT departments) and started a prospective database recording referrals at the outset. The clinic was effectively offering a new service to the "Oxford region" (a geographic area with a population of approximately 2.7 million).

Data collected included referral source, diagnosis, and management decision. We have compared our findings with data from the national and international literature. The chi-square test was performed to establish whether any change in practice was statistically significant.

\section{Results}

\section{Incidence of Acoustic Neuroma Diagnosis}

Between 1990 and 2009, a total of 1,308 patients were managed at the OSBC, of which $86 \%(1,090)$ had benign disease and $14 \%$ (173) malignant disease. Of all 1,308 patients, $55 \%$ (714) had a diagnosis of acoustic neuroma, though complete data regarding management was available for only 703 of the 714 . - Fig. 1

The majority of acoustic neuroma patients were referred by other ENT surgeons (85\%, 604 out of 714), suggesting that the vast majority of these patients are diagnosed by referral to an ENT department with their initial symptoms of sensorineural hearing loss/tinnitus/imbalance. Those referred by other physicians (e.g., neurologists, maxillofacial surgeons) were predominantly patients with larger tumors presenting with trigeminal/brainstem-related symptoms.

\section{Management Decisions}

To compare the OSBC management decisions with national practice, we first examined our management decisions from the year 2001 and compared these with the only published United Kingdom national survey/audit of practice, conducted in 2001 (-Fig. 2). ${ }^{2}$ This demonstrated a much larger proportion of patients at the OSBC being treated with GK radiotherapy with less being treated surgically. - Fig. 2

To determine the significance of this difference in practice, we performed a chi-square analysis comparing all patients who underwent active treatment, excluding patients in the observation groups. This difference between groups in the proportion of patients treated with each modality is significant with a p value $<0.001$, as seen in - Table 1 and - Table 2 (-Fig. 3).

To quantify our change in practice at the OSBC, we compared practice in the first 10 years (1990 to 1999) with that of the second 10 years. This shows a large difference in the proportion of patients treated with radiotherapy versus surgery during the first and second decades at OSBC (- Fig. 4)

To determine if this change in practice is significant, we performed a chi- square analysis of all patients actively treated with either surgery or radiotherapy, as seen in - Table 3.

Very recently there has been a further national audit of practice by the British Skull Base Society (unpublished data) for the year 2010 to 2011, which allows us to reexamine how the OSBC practice compares to national practice today (-Fig. 5). Again, the difference in the proportion of patients being treated with radiotherapy versus surgery between the two groups is significant $(p<0.001)$. It should be noted that this most recent audit (including the OSBC results displayed here) includes only new diagnoses of sporadic acoustic neuromas and excludes any patients with neurofibromatosis type 2 . In addition, out of the 33 units invited to participate, only 14 contributed, meaning there may be a significant selection bias which should be borne in mind when interpreting these results ${ }^{3}$ (-Fig. 5). 


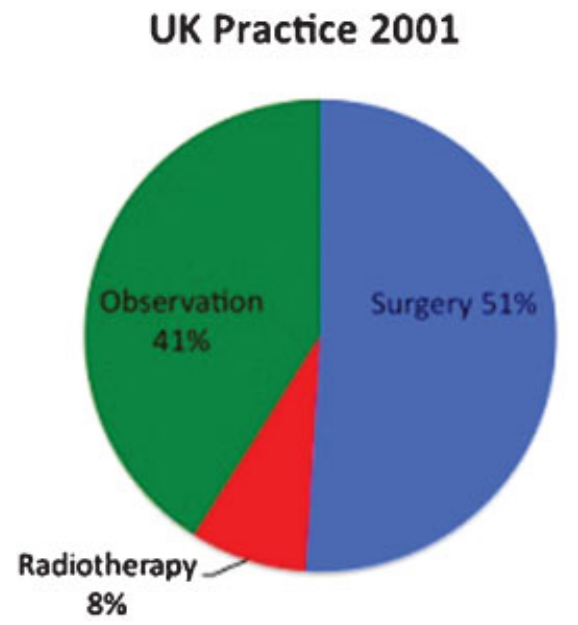

\section{Oxford Skull Base Clinic 2001}

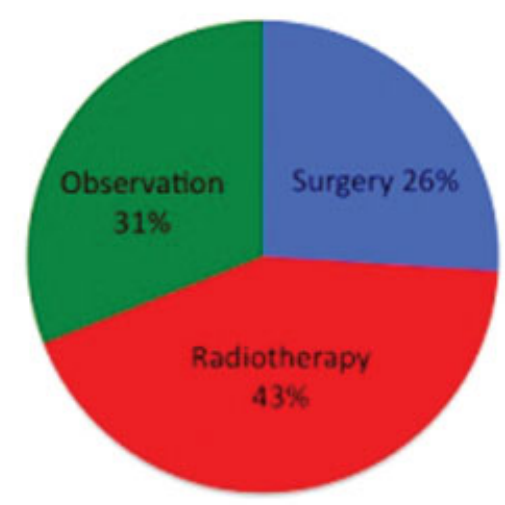

Fig. 2 Comparison of the Oxford Skull Base Clinic with data from a United Kingdom audit of practice from 2001 showing the proportion of patients managed with observation versus surgery versus radiotherapy.

Table 1 Number of Patients Actively Treated with Either Surgery or Radiotherapy Using Data from the OSBC and National Survey Data

\begin{tabular}{|l|l|l|l|}
\hline & $\begin{array}{l}\text { United Kingdom } \\
\text { practice 2001 }\end{array}$ & $\begin{array}{l}\text { OSBC } \\
\text { practice } \\
2001\end{array}$ & $\begin{array}{l}\text { Chi-square } \\
\text { test }\end{array}$ \\
\hline Surgery & $386 / 449$ & $10 / 27$ & \multirow{2}{*}{$p<0.001$} \\
\cline { 1 - 3 } Radiotherapy & $63 / 449$ & $17 / 27$ & \\
\hline
\end{tabular}

Abbreviation: OSBC, Oxford Skull Base Clinic

Table 2 Management Decision for All Patients with Acoustic Neuromas for Each Year During the 20-year Oeriod at Oxford Skull Base Clinic (OSBC)

\begin{tabular}{|l|l|l|l|l|}
\hline Year & Surgery & Radiotherapy & Observation & Total \\
\hline 1990 & 2 & 0 & 1 & 3 \\
\hline 1991 & 1 & 0 & 1 & 2 \\
\hline 1992 & 1 & 0 & 1 & 2 \\
\hline 1993 & 2 & 0 & 1 & 3 \\
\hline 1994 & 7 & 1 & 16 & 24 \\
\hline 1995 & 12 & 0 & 11 & 23 \\
\hline 1996 & 13 & 0 & 25 & 38 \\
\hline 1997 & 25 & 1 & 10 & 36 \\
\hline 1998 & 22 & 4 & 2 & 28 \\
\hline 1999 & 21 & 6 & 25 & 52 \\
\hline 2000 & 4 & 18 & 9 & 31 \\
\hline 2001 & 10 & 17 & 12 & 39 \\
\hline 2002 & 10 & 15 & 15 & 40 \\
\hline 2003 & 14 & 13 & 30 & 57 \\
\hline 2004 & 15 & 19 & 24 & 58 \\
\hline 2005 & 10 & 21 & 13 & 44 \\
\hline
\end{tabular}

Table 2 (Continued)

\begin{tabular}{|l|l|l|l|l|}
\hline Year & Surgery & Radiotherapy & Observation & Total \\
\hline 2006 & 8 & 30 & 36 & 74 \\
\hline 2007 & 11 & 19 & 17 & 47 \\
\hline 2008 & 11 & 14 & 23 & 48 \\
\hline 2009 & 10 & 20 & 24 & 54 \\
\hline Total & $209(29.7 \%)$ & $198(28.2 \%)$ & $296(42.1 \%)$ & 703 \\
\hline
\end{tabular}

\section{Discussion}

When we look at the variation in annual referral numbers (-Fig. 1), it can be seen that during the first 10 years of the 20year period there was a gradual increase in the number of referrals of patients with a diagnosis of acoustic neuroma, mainly due to an increased number of referrals from local clinicians for what was effectively a new "combined" service (as opposed to an increase in diagnoses in the referral catchment area).

The second 10-year period demonstrates a consistent high volume of new acoustic neuroma referrals with an average of 48.1 per year (range 37 to 69). Despite this, - Fig. 3 shows that over the same 20 -year period, the number of patients treated surgically did not continue to rise. In fact the past 10 years show a reduced proportion of patients treated surgically compared with preceding years.

The management of acoustic neuromas internationally has changed significantly over recent decades. Historically, the mainstay of treatment was surgical with the primary aim being to eradicate disease. Subsequently many surgeons have moved toward the primary aim being preservation of function (e.g., facial nerve), accepting the possibility of subtotal excision. Interestingly, this more conservative approach was initially suggested by Cushing himself in 1917. ${ }^{4}$

The ability to accurately measure tumor size with the use of MRI has allowed for the identification of smaller tumors, 


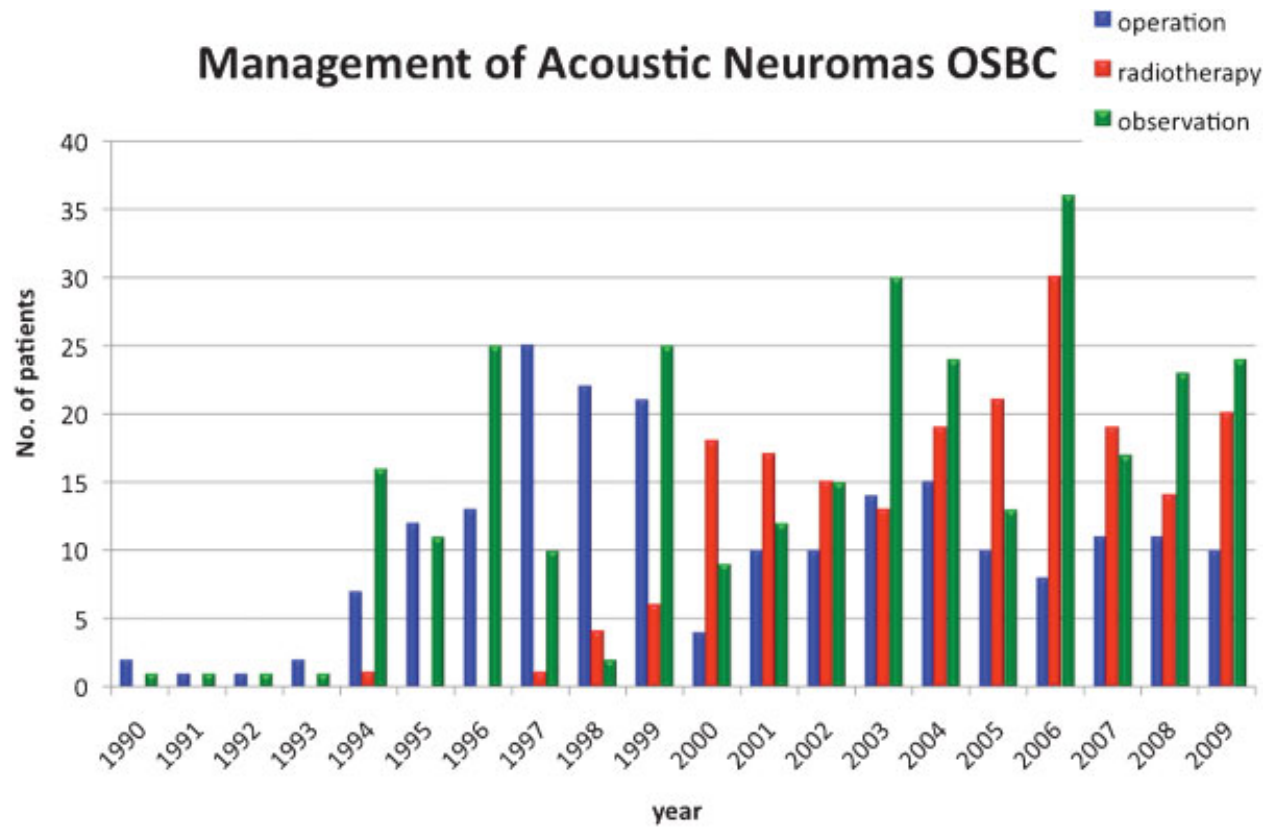

Fig. 3 Number of patients treated with each modality per year over the 20-year period at the Oxford Skull Base Clinic.
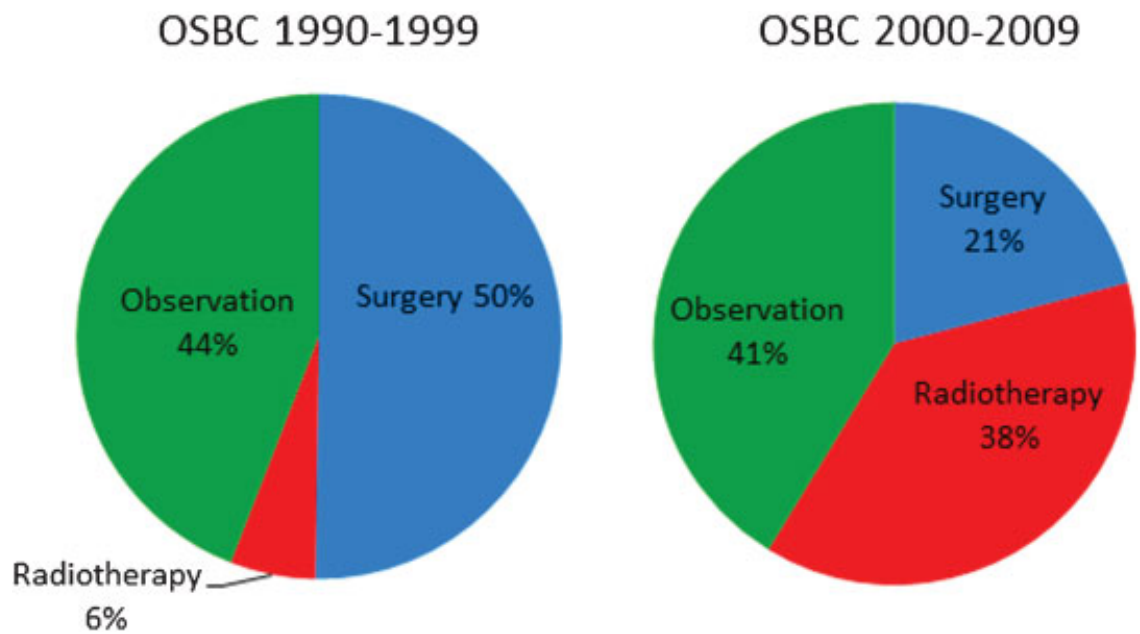

Fig. 4 Proportion of patients treated with each treatment modality over the two time periods of 1990 to 1999 and 2000 to 2009 at Oxford Skull Base Clinic.

monitoring of size, and new knowledge acquisition as to the natural history of this disease. Knowing that many acoustic tumors will not grow, or may even involute, has given rise to the management option of observation. Indeed, there is increasing data to help stratify patients at the time of diagnosis as to which are more likely to have further tumor

Table 3 Management Decision for All Patients Who Underwent Active Treatment (e.g., not observation/serial scanning) at Oxford Skull Base Clinic During these Two Time Periods

\begin{tabular}{|l|l|l|l|}
\hline & $\begin{array}{l}\text { OSBC } \\
1990-1999\end{array}$ & $\begin{array}{l}\text { OSBC } \\
\text { 2000-2009 }\end{array}$ & $\begin{array}{c}\text { Chi-square } \\
\text { test }\end{array}$ \\
\cline { 1 - 3 } Surgery & $106 / 118$ & $103 / 289$ & $<0.001$ \\
\cline { 1 - 3 } Radiotherapy & $12 / 118$ & $186 / 289$ & \\
\hline
\end{tabular}

growth. ${ }^{5}$ Other studies clearly show an increase in the number of patients treated with initial observation in the last decade when compared with previous decades.

Expert opinion during the 1990s was that "The correct treatment for the majority of patients with a small acoustic neuroma is prompt removal by an experienced surgical team"6. In addition, in 1991 in the United States, the National Institutes of Health issued a statement recommending surgery for all patients (with acoustic neuromas) who were willing and able to undergo an operation. ${ }^{6}$ With new published data showing the natural history of this disease, we now know that a proportion of these can be observed and may never need any further treatment.

Data from the United Kingdom also support the increase in the use of conservative management, with a paper from Manchester showing that two thirds of patients with acoustic 


\section{British Skull Base Society Audit 2011 Oxford Skull Base Clinic 2011}
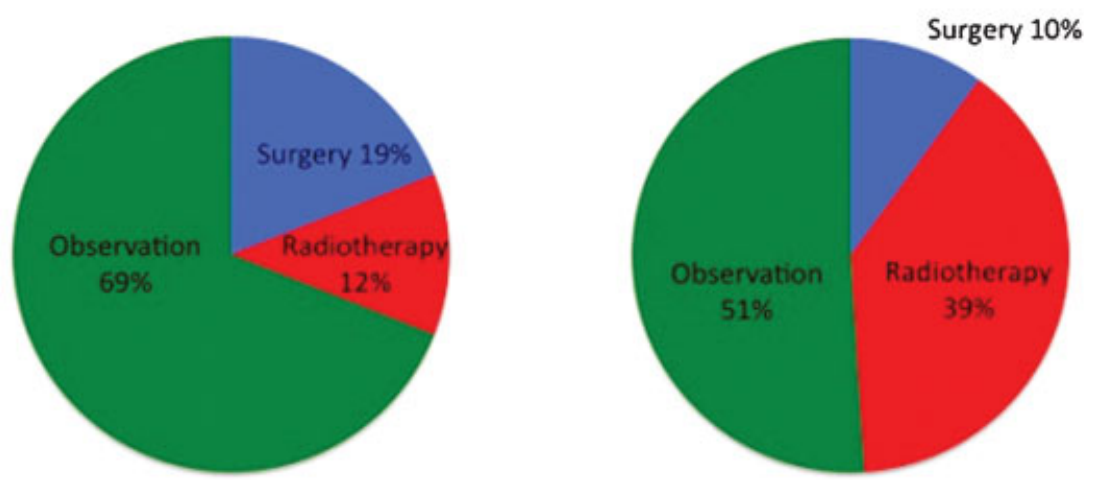

Fig. 5 Comparison of the Oxford Skull Base Clinic in 2011 with data from British Skull Base Society audit from 2011 showing the proportion of patients managed with observation versus surgery versus radiotherapy.

neuromas who were managed conservatively did not undergo any further growth. ${ }^{7}$ This and similar papers also provide new information on factors that can be used to stratify patients at increased risk of further growth, such as extracanalicular more than intracanalicular, intracranial (extracanalicular) component with diameter $>20 \mathrm{~mm}$, and young age. $^{7,8}$

The introduction of GK/stereotactic radiosurgery has been met with varying degrees of enthusiasm since its introduction. Criticisms of this treatment modality identify the failure to remove disease (as its aim is to arrest growth). This does, however, seem less of a drawback in our current working environment, as subtotal surgical resection becomes a more accepted technique to preserve facial nerve function. In addition, there is a lack of long-term follow-up data for this treatment modality, and there are therefore concerns regarding subsequent late regrowth. Despite this, proponents have published good results presenting it as a safe and effective alternative treatment to surgery. Direct comparison of radiosurgery with microsurgery is difficult due to the heterogeneity of studies with differing outcome criteria and data reporting. Certainly there are no randomized controlled trials comparing these two modalities, which would give clear guidelines as to best practice.

Reviewing the literature for evidence of trends in current practice to compare with our own, we see significant variations. A study from Johns Hopkins looking at the trends in acoustic neuroma management between 1997 and 2007 gives overall figures for treatment with surgery versus stereotactic radiotherapy versus observation as $75 \%, 3.6 \%$, and $21 \%$ respectively. The authors of this study also comment that over the 10-year period there was an increase in the proportion of patients treated with stereotactic radiotherapy or observation. This study may underestimate the number of patients who had radiotherapy, as they excluded 185 patients who did not return for subsequent management. ${ }^{9}$

Another series from a private neurotology practice in the United States describes trends in management of 614 patients with acoustic neuromas over the period 1990 to 2005. Divided into three periods-1990 to 1995,1995 to 2000 , and 2000 to 2005-the proportion of patients treated surgi- cally changed from $80 \%$ to $86 \%$ to $59 \%$. Whereas the proportion of patients managed with observation increased from $18 \%$ to $12 \%$ to $37 \%$ and the proportion of patients treated with stereotactic radiotherapy increased from $2 \%$ to $3 \%$ to $4 \% .{ }^{10}$

In contrast to these figures, a recent epidemiological study from the United States that identified 1,621 patients diagnosed with acoustic neuromas from a national tumor registry database in 2004 showed a much higher proportion of patients treated with radiation (21\%), with only $60 \%$ receiving surgical treatment. It may be that this variation is due to selection bias, as patients attending a private neurotology surgeon's clinic might already have a preference for surgical management when first presenting. ${ }^{11}$

Comparing the OSBC practice with what is known about acoustic neuroma management on a national level from the postal survey of United Kingdom ENT surgeons by Saeed et al, we see some major differences. ${ }^{2}$ In this survey, the average practice for 28 neurotologists in the United Kingdom managing 767 acoustic neuromas in 2001 shows the proportion of patients treated with either surgery, radiotherapy, or observation was $50 \%, 8 \%$, and $41 \%$, respectively, as opposed to the Oxford figures of $26 \%, 43 \%$, and $31 \%$. It is difficult to account for this apparent difference in practice at that time. It is possible that by surveying neurotologists directly, we are missing patients with acoustic neuromas who are referred directly for stereotactic radiosurgery and are underestimating the true number treated with this modality (as seen with the United States studies). However, we believe this source of error is less likely to arise in this United Kingdom survey (where the majority of units are run on a multidisciplinary basis) than in the United States.

A further explanation for this variation in practice could be that the Oxford region population may not be representative of the national population. Oxford is one of four centers treating patients with neurofibromatosis type 2 (NF2), and it could be argued that this could be a source of bias. However, in reality, the number of NF2 cases is very small, making up less than $3.5 \%$ of the total number of cases treated. Although these patients are probably more likely to receive radiotherapy, the impact of this on the overall results is minimal and unlikely to skew the results. 
Another geographical reason that may affect the use of radiotherapy is the proximity and ease of access to a good radiosurgical unit, which may not be the case for all regions.

It is interesting to observe that the British Skull Base Society national survey from $2011^{3}$ shows a marked change in practice when compared with the survey from $2001 .^{2}$ There seems to be a move away from surgical management (51\% to 19\%) and an increase in observation/serial scanning (41\% to $69 \%$ ), but also an increase in the number of patients having radiotherapy ( $8 \%$ to $12 \%$ ), which though small in absolute terms is a proportional increase of $33 \%$. It is reassuring to see that the change in practice seen over the past 20 years at the OSBC is in keeping with the changes in practice demonstrated (to a lesser extent) nationally and is reflective of a changing management philosophy.

Although not on the same scale as in our results, internationally there has been a move toward more patients being treated with stereotactic radiosurgery over the last 15 years. There are many reasons that have been suggested as the cause for this change. A simple explanation for this could be that over the past 20 to 30 years, acoustic neuromas are being diagnosed earlier and smaller due to improved referral pathways and more accurate imaging. A large epidemiological study from Denmark showed that the mean size of acoustic neuroma at diagnosis decreased from $30 \mathrm{~mm}$ in 1979 to $10 \mathrm{~mm}$ in $2004 .^{12}$ As it is generally accepted that large tumors are probably best treated surgically, the reducing proportion of large acoustic neuromas at presentation may account for the reduced proportion of patients being treated with microsurgery. (This is also supported by data from the most recent BSBS audit, which gives an average tumor size at diagnosis of $16.8 \mathrm{~mm}$, with $22 \%$ being intracanalicular). ${ }^{3}$ It is, however, unlikely that this is the sole explanation for the changing practice, and other factors should be considered.

Support for this change comes from an increasing volume of evidence from proponents of stereotactic radiosurgery, promoting it as an equal or superior treatment modality with good control rates (96\% and $94 \%$ at 3 and 7 years, respective$\left.\mathrm{ly}^{13,14}\right)$. Changes to patient awareness of treatment options, particularly with increasing use of the Internet, is thought to influence patient decision making. A study by Orabi et al showed that $24 \%$ of patients with acoustic neuromas obtained information from the Internet. ${ }^{15}$ Indeed, a study by Pogodzinski et al from the United States suggests that patients may have already decided on a treatment option when they choose to consult with either a microsurgeon or radiosurgeon. ${ }^{16}$ This direct access to a radiosurgeon is in contrast to United Kingdom practice, where patients are likely to be seen by an ENT, neurosurgeon, or both prior to referral to a radiosurgeon. Most research published comparing these two treatment modalities comes from large single-center patient cohorts where microsurgeons present results favoring surgery and radiosurgeons present equally persuasive results for radiosurgery. It is interesting, however, that in centers where the neurotological surgeons have become involved in providing radiation therapy (such as in the United States), the proportion of patients receiving this treatment has increased. ${ }^{10,11}$
As clinicians, we aim to always put the patient's best interests at the center of any clinical decision. It should be acknowledged that occasionally external (or even personal) factors may influence surgeons' decision making. Although difficult to measure, it is possible that financial incentive may explain some geographical differences in rates of microsurgery versus radiosurgery in the United States versus United Kingdom. Similarly, with centralization and subspecialization, the British Association of Otolaryngologists-Head and Neck Surgeons recommends that patients with acoustic neuromas be treated by a specialist team of a neurotologist and a neurosurgeon with sufficient caseload to maintain expertise. ${ }^{17}$ It is possible that surgeons may wish to protect their practice by maintaining sufficient throughput of surgical cases. Interestingly, in the United Kingdom survey of practice, the four surgeons with the largest caseload ( $>50$ per year) had the highest rate of microsurgical removal and the lowest rate of referral for radiotherapy. ${ }^{2}$

Although there is no definitive evidence for one treatment modality over another, clinicians should nevertheless make patients aware of the various treatment options. Unfortunately, results from a pretreatment survey by the British Acoustic Neuroma Association (BANA), published in 2001, found that over $75 \%$ of patients did not feel they had their treatment options adequately explained to them. In fact, only 115 out of 472 patients had radiotherapy or radiosurgery discussed as a possible alternative to microsurgery. ${ }^{18}$ We believe, however, that if this national patient survey were to be repeated today, the percentage of patients having discussion of all treatment modalities would be much higher. It could be suggested that the relative affluence of the Oxford region (compared with the national average) might correspond to patients being better informed via available resources such as the internet and BANA. This increased awareness of all modalities of treatment may partly contribute to the explanation for the increased choice of radiotherapy.

It would be interesting to determine if this correlation was genuine when compared on a national level.

Overall, it is important to state that the changes we have observed in the management of acoustic neuromas at OSBC has not been a result of a conscious decision to change practice, but more an evolution of practice as a result of multiple factors. It is difficult to determine which elements are most important in influencing this change. In addition to more obvious clinical reasons, such as earlier detection with MRI and an increasing body of evidence to support radiotherapy as an alternative treatment option, we feel that changes to society and the doctor-patient relationship are important factors to consider.

The change in society and patients' attitudes may be in part due to improved access to information (and misinformation) via resources such as the Internet. Better-informed patients are expecting a greater degree of involvement in clinical decisions. Current practice is that all patients are informed of the natural history of the disease, the treatment options available, and the potential complications and outcomes of treatment. Patients are then given help and guidance in making their own decision regarding management. In effect, 
perhaps patients have been voting with their feet, with an increasing number choosing radiotherapy.

Alongside this has been a change in doctors attitudes. This is well described by the Royal College of Physicians Working Party document, "Doctors in society. Medical professionalism in a changing world" 19 :

...because so much of medicine's unpredictability calls for wisdom as well as technical ability, doctors are vulnerable to the charge that their decisions are neither transparent nor accountable. In an age where deference is dead and league tables are the norm, doctors must be clearer about what they do and why they do it....

Clinical management decisions must therefore be entirely transparent, incorporating best available evidence with clinical expertise, both tailored to the individual patient. When there is no definitive evidence of one treatment over another, patients should be made aware of the relative advantages and disadvantages and given the opportunity to choose for themselves.

Despite huge advances in the diagnosis and treatment of acoustic neuromas over the last century, one of the most difficult questions to answer remains that of when to operate and when not to operate (and give radiotherapy or just observe). It is interesting to note that Cushing acknowledged this as one of the greatest challenges over a century ago: "I would like to see the day when somebody would be appointed surgeon somewhere who had no hands, for the operative part is the least part of the work." (Harvey Cushing, letter to Dr Henry Christian, November 1911)

\section{Conclusion}

It seems clear that the past 20 years has seen significant changes in the diagnosis and treatment of acoustic neuromas, alongside an evolving management philosophy with increased patient awareness and involvement. There is no single best treatment for every patient with this condition, but what is clear is that patients should be fully informed of treatment options, and this should guide individual management decisions on a case-by-case basis. Surgery will remain crucial in the management of some patients with acoustic neuromas (usually those with the larger tumors where radiosurgery is recognized to be less appropriate), but using current trends to predict future practice would suggest that alternative nonmicrosurgical treatment may play an increasingly important role in the future.

\section{Acknowledgments}

Thanks to Patrick Axon, Consultant ENT Surgeon, for providing unpublished data from the British Skull Base Society Vestibular Schwannoma Audit 2011.

\section{References}

1 House WF. A history of acoustic tumor surgery. In: House WF, Luetje CM, eds. Acoustic Tumors. Baltimore: University Park Press; 1979:9-32

2 Saeed SR, Suryanarayanan R, Dezso A, Ramsden RT. Vestibular schwannoma management: current practice amongst UK otolaryngologists-time for a national prospective audit. Ann R Coll Surg Engl 2006;88(5):490-495

3 British Skull Base Society Vestibular Schwannoma Audit 2011. Unpublished - data provided by Patrick Axon, Consultant ENT Surgeon, Addenbrooke's Hospital, Cambridge, UK

4 Cushing H. Tumours of the nervus acusticus and the syndrome of the cerebellopontine angle. Philadelphia: W.B. Saunders Co; 1917:1-295

5 Neuroma A. NIH Consens Statement 1991;9(4):1-24

6 Ramsden RT, Moffat DA. Intracanalicular acoustic neuromas: the case for early surgery. Clin Otolaryngol Allied Sci 1994; 19(1):1-2

7 Suryanarayanan R, Ramsden RT, Saeed SR, et al. Vestibular schwannoma: role of conservative management. J Laryngol Otol 2010;124(3):251-257

8 Ogawa K, Kanzaki J, Ogawa S, Yamamoto M, Ikeda S, Shiobara R. The growth rate of acoustic neuromas. Acta Otolaryngol Suppl 1991;487:157-163

9 Tan M, Myrie OA, Lin FR, et al. Trends in the management of vestibular schwannomas at Johns Hopkins 1997-2007. Laryngoscope 2010;120(1):144-149

10 Chen DA. Acoustic neuroma in a private neurotology practice: trends in demographics and practice patterns. Laryngoscope 2007;117(11):2003-2012

11 Gal TJ, Shinn J, Huang B. Current epidemiology and management trends in acoustic neuroma. Otolaryngol Head Neck Surg 2010;142(5):677-681

12 Stangerup SE, Tos M, Thomsen J, Caye-Thomasen P. True incidence of vestibular schwannoma? Neurosurgery 2010;67(5): 1335-1340, discussion 1340

13 Pollock BE. Vestibular schwannoma management: an evidencebased comparison of stereotactic radiosurgery and microsurgical resection. Prog Neurol Surg 2008;21:222-227

14 Pollock BE, Driscoll CL, Foote RL, et al. Patient outcomes after vestibular schwannoma management: a prospective comparison of microsurgical resection and stereotactic radiosurgery. Neurosurgery 2006;59(1):77-85, discussion 77-85

15 Orabi AA, D'Souza AR, Walsh RR, Irving RM. The influence of the Internet on decision making in acoustic neuroma. J Laryngol Otol 2005;119(10):806-809

16 Pogodzinski MS, Harner SG, Link MJ. Patient choice in treatment of vestibular schwannoma. Otolaryngol Head Neck Surg 2004; 130(5):611-616

17 British Association of Otorhinolaryngologists - Head and Neck Surgeons Clinical Practice Advisory Group. Clinical Effectiveness Guidelines Acoustic Neuroma (Vestibular Schwannoma). BAOHNS document 5; 2002.

18 British Acoustic Neuroma Association. Pre-Treatment Survey. Published March 2001. Available at: http://www.bana-uk.com/ index.php?option=com_content\&task=view\&id=107\&Itemid=11. Accessed March 21, 2013

19 Working Party of the Royal College of Physicians . Doctors in society. Medical professionalism in a changing world. Clin Med 2005;5(6, Suppl 1):S5-S40 